\title{
Birth of a healthy baby after preimplantation genetic diagnosis in a carrier of mucopolysaccharidosis type Il: The first case in Korea
}

\author{
Duck Sung Ko' ${ }^{1}$, Sun-Hee Lee', Chan Woo Park², Chun Kyu Lim ${ }^{1}$ \\ 'Laboratory of Reproductive Medicine and 'Department of Obstetrics and Gynecology, Cheil General Hospital and Women's Healthcare Center, Seoul, \\ Korea
}

Mucopolysaccharidosis type II (MPS II) is a rare X-linked recessive lysosomal storage disease caused by mutation of the iduronate-2-sulfatase gene. The mutation results in iduronate-2-sulfatase deficiency, which causes the progressive accumulation of heparan sulfate and dermatan sulfate in cellular lysosomes. The phenotype, age of onset, and symptoms of MPS II vary; accordingly, the disease can be classified into either the early-onset type or the late-onset type, depending on the age of onset and the severity of the symptoms. In patients with severe MPS II, symptoms typically first appear between 2 and 5 years of age. Patients with severe MPS II usually die in the second decade of life although some patients with less severe disease have survived into their fifth or sixth decade. Here, we report the establishment of a preimplantation genetic diagnosis (PGD) strategy using multiplex nested polymerase chain reaction, direct sequencing, and linkage analysis. Unaffected embryos were selected via the diagnosis of a single blastomere, and a healthy boy was delivered by a female carrier of MPS II. This is the first successful application of PGD in a patient with MPS II in Korea.

Keywords: Mucopolysaccharidosis II; Multiplex polymerase chain reaction; Preimplantation genetic diagnosis

\section{Introduction}

Mucopolysaccharidosis type II (MPS II; OMIM \#309900), also known as Hunter syndrome, is a rare X-linked recessive disease. MPS II is caused by mutation of the IDS gene, which is located on chromosome Xq28. The mutation results in a deficiency of the enzyme iduronate2-sulfatase (IDS). This deficiency leads to the progressive accumulation of the glycosaminoglycans dermatan sulfate and heparan sulfate in the lysosomes of nearly all cell types, tissues, and organs, contributing to the signs and symptoms of MPS II. The most common clinical

Received: February 27, 2019 · Revised: June 24, 2019 · Accepted: October 10, 2019 Corresponding author: Chun Kyu Lim

Laboratory of Reproductive Medicine, Cheil General Hospital and Women's Healthcare Center, 17 Seoae-ro 1-gil, Jung-gu, Seoul 04619, Korea Tel: +82-2-2000-7590 Fax:+82-2-2265-5621 E-mail:seungzzang@paran.com

This is an Open Access article distributed under the terms of the Creative Commons Attribution Non-Commercial License (http://creativecommons.org/licenses/by-nc/4.0/) which permits unrestricted non-commercial use, distribution, and reproduction in any medium, provided the original work is properly cited. features of MPS II include coarse facial features, stiff joints, short stature, skeletal deformities, hepatosplenomegaly, cardiovascular and respiratory disorders, developmental delay, and mental retardation [1]. This disease generally affects males almost exclusively, and only a few cases of female patients with MPS II have been described to date [2,3]. The worldwide incidence of MPS II is one in 100,000 to one in 170,000 male births [4]. To date, more than 550 pathogenic variants have been described, the majority of which are point mutations or small deletions (HGMD Professional 2016.2; www.hgmd.cf.ac.uk). In many countries, including South Korea, IDS gene mutations in MPS II patients have been extensively studied $[5,6]$.

Preimplantation genetic diagnosis (PGD) is a well-established reproductive option for couples at risk of transmitting known genetic disorders to their offspring. To our knowledge, no report has been published detailing the birth of a healthy baby after PGD of MPS II in Korea. Therefore, we describe the establishment of a PGD strategy in an MPS II carrier, the successful application of PGD, and the birth of a 
healthy boy after PGD of MPS II for the first time in Korea.

\section{Case report}

\section{Patient history}

A female carrier of the c.998C $>$ T mutation of the IDS gene visited our hospital in 2016 to undergo PGD. The woman was 36 years old and had experienced the termination of a pregnancy of a male fetus with MPS II in 2013. Her mother and one of her two sisters were carriers of the mutation, and she had a nephew with MPS II (Figure 1). The couple desired to have a healthy baby without the IDS mutation and therefore underwent $P G D$. The in vitro fertilization (IVF) and PGD procedures, as well as the limitations and accuracy of PGD, were explained to the couple, who signed an informed consent form for PGD.

\section{Preclinical studies}

Preclinical studies and lymphocyte validation were performed prior to the IVF cycle according to the recommendations of international guidelines on PGD $[7,8]$. DNA was extracted from peripheral blood from the couple and from the family members of the female carrier to confirm the presence of the mutation and determine the informative short tandem repeat (STR) markers for haplotype analysis. Five STR markers closely linked to the IDS gene were tested to avoid misdiagnosis due to allelic dropout (ADO; random non-amplification of one of the alleles) during the PGD procedures [9]. The single-cell multiplex nested polymerase chain reaction (PCR) strategy was established using a single lymphocyte. We performed PCR for the selected informative markers and the mutation site of the IDS gene. The established multiplex nested PCR strategy was applied to the preclinical set-up using single lymphocytes, and a clinical PGD cycle was conducted using blastomeres.

The outer and inner pairs of primers (forward and reverse) for the hemi-nested PCR amplification of sequences encompassing each mutation and the STR markers were designed using Primer 3 software (http://frodo.wi.mit.edu) and acquired. Five STR makers close to the IDS gene (DXS457, DXS7423, DXS8377, DXS1123, and DXS1193) were chosen according to the National Center for Biotechnology Information database (UCSC genome browser) for linkage and haplotype analysis. The primer sequences are reported in Table 1, and the results of the haplotype analysis are shown in Figure 1. Five STR markers located at both sides of the IDS gene were tested; among them, two were identified as semi-informative (DXS1123 and DXS1193).

\section{Establishment of PGD strategy}

PGD was performed by direct sequencing of the mutation site of the IDS gene and linkage analysis using the DXS1193 STR marker and the amelogenin gene as a sex marker. The first-round multiplex PCR was performed using outer primers for the mutation site and the two

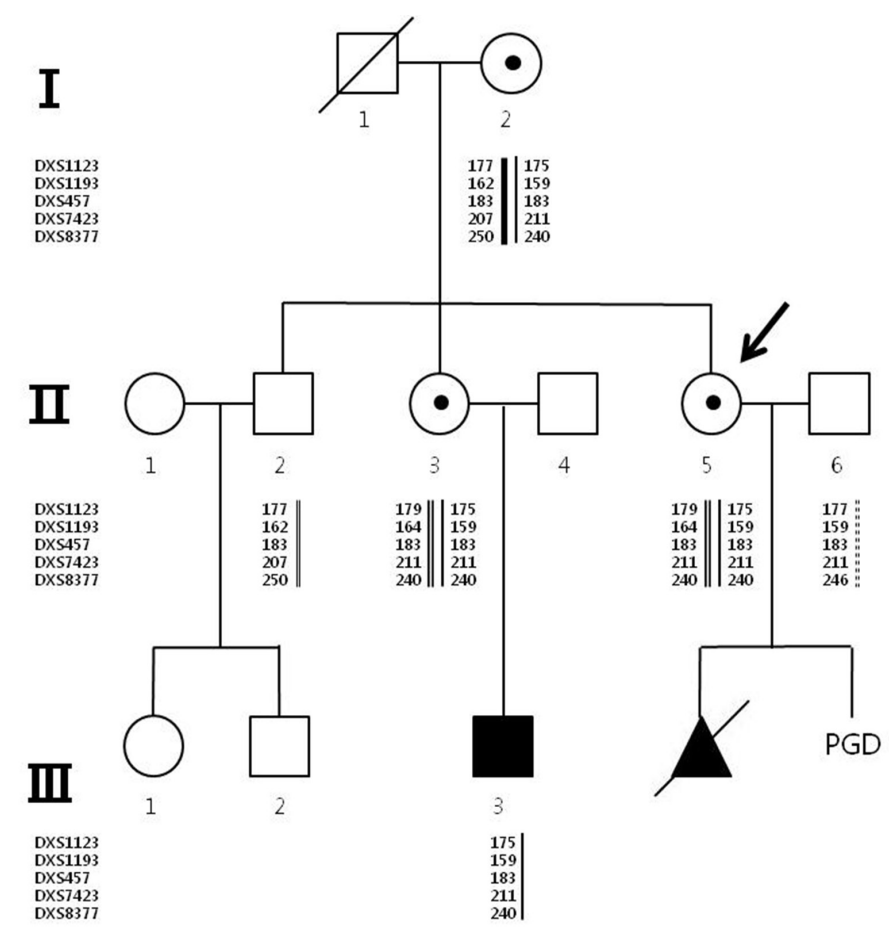

Figure 1. Pedigree and haplotype map of the family with mucopolysaccharidosis type II (MPS II). Squares and circles indicate male and female family members, respectively. The arrow indicates the proband, the black symbols represent those affected by MPS II, and the circles with the black circles inside them represent female carriers of MPS II. PGD, preimplantation genetic diagnosis. 
markers. The reaction mixture contained $200 \mu \mathrm{M}$ deoxyribonucleotide triphosphates (dNTP), ×1 PCR buffer (NeoTherm; GeneCraft, Muenster, Germany), 1 U of DNA polymerase (NeoTherm, Gene Craft), and $0.2 \mu \mathrm{M}$ each of the forward and reverse primers in a final volume of $30 \mu \mathrm{L}$. The first round of PCR was performed in a thermocycler (C1000; Bio-Rad, Hercules, CA, USA) using the following PCR protocol: 10 minutes at $94^{\circ} \mathrm{C} ; 25$ cycles of 30 seconds at $94^{\circ} \mathrm{C}, 30 \mathrm{sec}-$ onds at $60^{\circ} \mathrm{C}$, and 40 seconds at $72^{\circ} \mathrm{C}$; and finally 10 minutes at $72^{\circ} \mathrm{C}$. A second round of PCR was performed using $0.5 \mu \mathrm{L}$ of the product of the first round of PCR as template DNA. The reaction mixture contained $200 \mu \mathrm{M}$ dNTP, $\times 1$ NeoTherm PCR buffer, 1 U NeoTherm DNA polymerase, and $0.2 \mu \mathrm{M}$ each of the inner forward and reverse primers in a final volume of $30 \mu \mathrm{L}$. The second round of PCR was similarly performed in a C1000 thermocycler using the following PCR protocol: 10 minutes at $94^{\circ} \mathrm{C} ; 40$ cycles of 30 seconds at $94^{\circ} \mathrm{C}, 30$ seconds at $62^{\circ} \mathrm{C}$, and 40 seconds at $72^{\circ} \mathrm{C}$; and finally 10 minutes at $72^{\circ} \mathrm{C}$. The amplification of the inner products of the mutation site and the STR markers was verified by agarose gel electrophoresis. A DNA sequencing reaction for direct detection of the mutation was performed using the BigDye Terminator v3.1 Cycle Sequencing Kit (Applied Biosystems, Forest City, IA, USA). Analysis of the sequencing reaction product and amplified STR markers was performed using an ABI $3500 \mathrm{Ge}$ -

Table 1. Primer sequences used in the preclinical validation of the protocol and the preimplantation genetic diagnosis

\begin{tabular}{|c|c|c|c|}
\hline Name & Outer PCR & Inner PCR & Size (bp) \\
\hline \multirow[t]{2}{*}{ IDSExon7 } & F: 5'-GCCTCTTGAGTGCTTTGGAC-3' & F: 5'-GCCTCTTGAGTGCTTTGGAC-3' & 361 \\
\hline & R: $5^{\prime}$-TACAAGAGCTCTGCCACACA-3' & R: $5^{\prime}$-ACCCACACCTATCCGTCAAG-3' & \\
\hline \multirow[t]{2}{*}{ DXS1123 } & F: 5'-TGCCTAAATGTTCGCAAGCCCATTC-3 & F: 5'-TGCCTAAATGTTCGCAAGCCCATTC-3' & 163 \\
\hline & R: $5^{\prime}$-CATCCACACTCTCCCCAAAT-3' & R: $f-5^{\prime}$-ACAAACAGCTGCCTCCTAGAAACCC-3' & \\
\hline \multirow[t]{2}{*}{ DXS1193 } & F: $5^{\prime}$-AATTCTGACTCTGGGGC-3' & F: $n-5^{\prime}$-ACTCTCCTCTCCTGGCTTCC-3' & 170 \\
\hline & R: 5'-TTACCCACTTCCCCACTGTC-3' & R: 5'-TTACCCACTTCCCCACTGTC-3' & \\
\hline \multirow[t]{2}{*}{ DXS457 } & F: 5'-TAGCCTGCTAGCCTGCTTGT-3' & F: $h-5^{\prime}$-CCTGACAATCACATAAGCTA-3' & 117 \\
\hline & R: $5^{\prime}$-CAGATCAGGCATGCTCAAAC-3' & R: $5^{\prime}$-CAGATCAGGCATGCTCAAAC-3' & \\
\hline \multirow[t]{2}{*}{ DXS8377 } & F: 5'-GGCCCCAGCCTACATCTAC-3' & F: $f-5^{\prime}-C A C T T C A T G G C T T A C C A C A G-3^{\prime}$ & 246 \\
\hline & R: 5'-GACCTTTGGAAGCTAGTGT-3' & R: 5'-GACCTTTGGAAGCTAGTGT-3' & \\
\hline \multirow[t]{2}{*}{ DXS7423 } & F: 5'-TGCGAGCCCACTCTTTCTAT-3' & F: $n-5^{\prime}-$ GTCTTCCTGTCATCTCCCAAC-3' & 221 \\
\hline & R: 5'-TGGCCTTTGTCTCCAGTACC-3' & R: 5'-GTCTTCCTGTCATCTCCCAAC-3' & \\
\hline \multirow[t]{2}{*}{ Amelogenin } & F: 5'-TGGGCACCCTGGTTATATCAACT-3' & F: $f-5^{\prime}-C C C T G G G C T C T G T A A A G A A T A G T G-3^{\prime}$ & 114 \\
\hline & R: 5'-AGGCCAACCATCAGAGCTTAAACT-3' & R: 5'-AGGCCAACCATCAGAGCTTAAACT-3' & \\
\hline
\end{tabular}

PCR, polymerase chain reaction; $F$, forward primer; $R$, reverse primer; $f, 6$ FAM $5^{\prime}$-labeled primer; $n$, NED $5^{\prime}$-labeled primer; $h$, HEX $5^{\prime}$-labeled-primer.

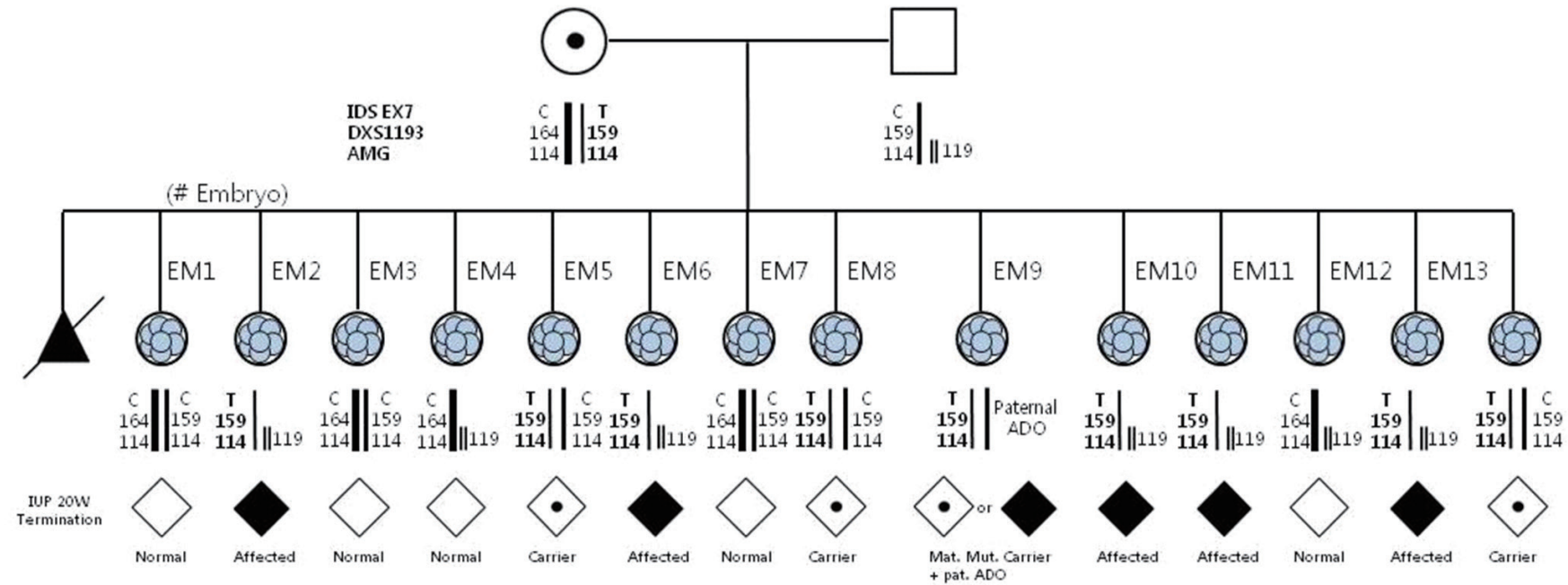

Figure 2. Preimplantation genetic diagnosis of mucopolysaccharidosis type II (MPS II). The bold lines represent the normal alleles for the IDS gene. Bold letters or numbers represent the IDS gene mutation in each family member or embryo. The numbers represent the size of polymerase chain reaction products in base pair. The directly sequenced nucleotide at position c.998 in the IDS gene is indicated by C (cytosine) or T (thymidine), corresponding to a normal or a mutated nucleotide, respectively. AMG, amelogenin for sex identification; mat, maternal; mut, mutation; pat, paternal; ADO, allelic dropout. 
netic Analyzer (Applied Biosystems). The data were then analyzed using SeqA6 software or GeneMapper (Applied Biosystems). After its establishment, the PGD strategy was validated using 40 single isolated lymphocytes. The amplification failure rate and the ADO rate for each PCR amplicon were estimated. The amplification failure rate was $0 \%$ (0 of 40) for exon 7 of the IDS gene and 5\% (1 of 40) for DXS1193. The ADO rate was 5\% (1 of 40) for the c.998C > T mutation and 5\% (1 of 40) for DXS1193.

\section{Ovarian stimulation, IVF, and PGD}

Controlled ovarian hyperstimulation was performed using a gonadotropin-releasing hormone agonist, recombinant follicle-stimulating hormone, and human chorionic gonadotropin. Twenty-one cumulus-oocyte-corona complexes were retrieved. Among them, 21 oocytes were in metaphase II, and sperm was injected into the oocytes. Normal fertilization (including the appearance of two pronuclei and a second polar body) was observed in 14 oocytes. Two blastomeres were biopsied from each of 14 cleavage-stage embryos on day 3 after oocyte retrieval. Twenty-eight blastomeres were diagnosed using multiplex nested PCR for the genetic analysis. Thirteen embryos were successfully diagnosed. One embryo could not be diagnosed due to amplification failure of the IDS gene. Six embryos were diagnosed as unaffected, three as heterozygous, and five as affected (Figure 2). The total ADO and amplification rates were $3.6 \%$ (1/28) and 96.4\% (27/28), respectively. Two embryos (numbers 4 and 12) were transferred to the patient's uterus 4 days after oocyte retrieval. A singleton pregnancy was achieved. To confirm the PGD results, the prenatal diagnosis was made using amniocentesis at 16 weeks of gestation. The prenatal diagnosis confirmed that the fetus had an unaffected genotype without mutation in the IDS gene. A healthy boy was delivered by cesarean section at 38 weeks of gestation without any complications.

\section{Discussion}

MPS II is a variable progressive multisystemic disorder. The phenotypic spectrum of MPS II is also variable. Although enzyme replacement therapy (ERT) is currently available for the treatment of MPS II, ERT only moderately improves its symptoms and does not improve neurological features in a clinically meaningful manner. Since the improvement of symptoms through ERT or other treatment is very minor, the best treatment for MPS II is the prevention of its inheritance, which is currently possible only via PGD. This is also true for some genetic diseases other than MPS II although PGD is not possible for all genetic diseases. This is the first report in Korea on the establishment and successful application of a reliable PGD strategy for MPS II.

Altarescu et al. $[9,10]$ reported the first successful application of
PGD of MPS II. According to that study, three couples underwent several cycles of PGD and successfully delivered healthy babies. Polar bodies were biopsied and used for diagnosis in two of the three couples. Polar body biopsy has several advantages in diagnosis, but its use is gradually decreasing for several reasons [11]. Therefore, we instead biopsied blastomeres from cleavage-stage embryos. Although blastomere biopsy is more invasive than polar body biopsy, we chose to analyze two blastomeres to ensure a more accurate analysis. Indeed, the PGD guidelines published by the European Society of Human Reproduction and Embryology recommend the analysis of two blastomeres to reduce the ADO rate when PGD is performed using blastomeres [7]. Thus, it was possible for us to reduce the amplification failure and ADO rate by analyzing two blastomeres and performing PGD with high accuracy.

We selected five STR markers located near mutation sites on the IDS gene and tested whether these markers could be used for PGD. However, we could not identify fully informative markers; instead, we identified only two semi-informative markers, DXS1123 and DXS1193. The ADO rate of DXS1123 was too high to be used as a marker for multiplex PCR. The maternal mutant allele for DXS1193 was the same size as the paternal normal allele. Therefore, it was possible that carrier embryos or affected embryos could not be distinguished from healthy embryos if the maternal mutant allele failed to amplify. When PGD was performed, only the maternal mutant allele was identified in embryo 9. We could not determine this embryo's genotype. In summary, we used a semi-informative marker for PGD because we could identify only semi-informative markers, and this meant that we could not determine the genotype of one embryo. Nonetheless, it is ideal to select and use fully informative markers in order to reach an accurate diagnosis.

When a woman is pregnant and she or her partner has a genetic disease, they face risks associated with methods of invasive prenatal diagnosis, such as chorionic villus sampling and amniocentesis. When a fetus is identified as having a genetic disease, the couple must decide whether to terminate the pregnancy. The decision is very difficult and traumatic to the couple. If a couple decides to give birth to the baby rather than terminating the pregnancy, they are likely to encounter many economic, medical, and physical difficulties. Therefore, many couples usually decide to terminate; however, termination of pregnancy may similarly be very traumatic. PGD is currently the only medical option for couples with genetic diseases to avoid a pregnancy with an affected baby and the subsequent possibility of termination. According to the PGD Consortium data collected by the European Society of Human Reproduction and Embryology, there have been 45,163 reported IVF cycles with PGD, 8,765 clinical pregnancies, and 7,134 deliveries between January 1999 and December 2012 since the first birth of a healthy baby using PGD in 1990 
[12]. The use of PGD leads to the birth of healthy babies.

However, many challenges must be overcome to achieve the highest possible levels of accuracy and reliability when single cells are analyzed in PGD. The risk of PGD includes all of the standard risks associated with IVF cycles (i.e., medication reaction, ovarian hyperstimulation, and higher frequency of chromosomal abnormalities) as well as the risks unique to the embryo testing procedure (i.e., affecting the developmental potential of embryos, false-positive results due to mosaicism, and genetic diagnostic error). PCR procedures using single cells, which are essential for the PGD of monogenic disorders, are extremely vulnerable to contamination by extraneous DNA and to the occurrence of ADO. These technical difficulties might lead to misdiagnosis in DNA amplification-based PGD. The main concern with PGD is the high possibility of misdiagnosis, mainly due to ADO and contamination. ADO is an extreme form of preferential amplification in which one of the two alleles fails to amplify. Under these circumstances, hemizygous loci result in complete absence of a PCR product. Thus, the highest possible accuracy for PGD can only be achieved when the diagnosis is confirmed for both noninheritance of the mutated allele(s) and presence of the wild allele(s). Multiplexing PCR for the mutation(s) with linked informative polymorphic STR markers has been shown to be quite helpful for the detection of both contamination and ADO. In this study, we established a reliable PGD strategy for MPS II. This is the first case report describing the establishment of a reliable PGD method and the live birth of a healthy baby after PGD in a Korean patient carrying the IDS gene mutation that causes MPS II.

\section{Conflict of interest}

No potential conflict of interest relevant to this article was reported.

\section{ORCID}

$\begin{array}{ll}\text { Duck Sung Ko } & \text { https://orcid.org/0000-0002-3914-4349 } \\ \text { Sun-Hee Lee } & \text { https://orcid.org/0000-0002-8270-1809 } \\ \text { Chan Woo Park } & \text { https://orcid.org/0000-0002-2564-6292 }\end{array}$

\section{Author contributions}

Conceptualization: DSK, CKL. Data curation: DSK, SHL, CWP. Formal analysis: DSK, SHL. Methodology: DSK, CKL. Project administration: CWP, CKL. Visualization: SHL, CWP, CKL. Writing - original draft: DSK. Writing - review \& editing: CKL.

\section{References}

1. Neufeld EF, Muenzer J. The mucopolysaccharidoses. In: Scriver CR, Beaudet AL, Valle D, Sly WS, editors. The metabolic and molecular bases of inherited disease. 8th ed. New York: McGrawHill; 2001. p. 3421-52.

2. Tuschl K, Gal A, Paschke E, Kircher S, Bodamer OA. Mucopolysaccharidosis type II in females: case report and review of literature. Pediatr Neurol 2005;32:270-2.

3. Sohn YB, Kim SJ, Park SW, Park HD, Ki CS, Kim CH, et al. A mother and daughter with the p.R443X mutation of mucopolysaccharidosis type II: genotype and phenotype analysis. Am J Med Genet A 2010;152:3129-32.

4. Baehner F, Schmiedeskamp C, Krummenauer F, Miebach E, Bajbouj M, Whybra C, et al. Cumulative incidence rates of the mucopolysaccharidoses in Germany. J Inherit Metab Dis 2005;28: 1011-7.

5. Sohn YB, Ki CS, Kim CH, Ko AR, Yook YJ, Lee SJ, et al. Identification of 11 novel mutations in 49 Korean patients with mucopolysaccharidosis type II. Clin Genet 2012;81:185-90.

6. Cho SY, Sohn YB, Jin DK. An overview of Korean patients with mucopolysaccharidosis and collaboration through the Asia Pacific MPS Network. Intractable Rare Dis Res 2014;3:79-86.

7. Preimplantation Genetic Diagnosis International Society (PGDIS). Guidelines for good practice in PGD: programme requirements and laboratory quality assurance. Reprod Biomed Online 2008; 16:134-47.

8. Harton GL, De Rycke M, Fiorentino F, Moutou C, SenGupta S, Traeger-Synodinos J, et al. ESHRE PGD consortium best practice guidelines for amplification-based PGD. Hum Reprod 2011;26: 33-40.

9. Altarescu G, Renbaum P, Eldar-Geva T, Brooks B, Varshaver I, Avitzour $\mathrm{M}$, et al. Preventing mucopolysaccharidosis type II (Hunter syndrome): PGD and establishing a Hunter $(46, \mathrm{XX})$ stem cell line. Prenat Diagn 2011;31:853-60.

10. Altarescu G, Beeri R, Eiges R, Epsztejn-Litman S, Eldar-Geva T, Elstein $\mathrm{D}$, et al. Prevention of lysosomal storage diseases and derivation of mutant stem cell lines by preimplantation genetic diagnosis. Mol Biol Int 2012;2012:797342.

11. Cimadomo D, Capalbo A, Ubaldi FM, Scarica C, Palagiano A, Canipari $\mathrm{R}$, et al. The impact of biopsy on human embryo developmental potential during preimplantation genetic diagnosis. Biomed Res Int 2016;2016:7193075.

12. De Rycke M, Goossens V, Kokkali G, Meijer-Hoogeveen M, Coonen E, Moutou C. ESHRE PGD Consortium data collection XIV-XV: cycles from January 2011 to December 2012 with pregnancy follow-up to October 2013. Hum Reprod 2017;32:1974-94. 